\title{
Thin-Plate Spline Approximation for Image Registration
}

\author{
Rainer Sprengel, Karl Rohr, H. Siegfried Stiehl \\ University of Hamburg, Fachbereich Informatik, Vogt-Kölln Str. 30, D-22527 Hamburg \\ email: sprengel@informatik.uni-hamburg.de
}

\begin{abstract}
The point-based registration technique of Bookstein [1] can be seen as an interpolation problem. With this technique an elastic transformation based on thin-plate splines is determined which maps the source and target landmarks exactly to each other. However, in real applications the positions of the landmarks can only be determined approximately. Therefore, in this case an interpolation scheme is inadequate and should be substituted by an approximation scheme to take into account the localization errors. In this paper, we describe an approach for extending Bookstein's method in this direction.
\end{abstract}

Keywords- Thin-Plate Splines, Regularization, Image Registration

\section{Thin-Plate Spline Interpolation}

The use of thin-plate spline interpolation as point-based elastic registration method of medical images was first proposed by Bookstein [1]. Below we briefly describe this method in the general context of $d$-dimensional images. The general problem can be stated as follows: Given two sets of $n$ landmarks $\mathbf{p}_{i}$ and $\mathbf{q}_{i}, i=1, \ldots, n$ in two image representations of dimension $d$. Within a suitable Hilbertspace $H^{d}$ of admissible functions find the transformation $\mathbf{u}$, which i) minimizes a given functional $J: H^{d} \rightarrow \mathbb{R}$ and ii) fulfills the interpolation conditions

$$
\mathbf{u}\left(\mathbf{p}_{i}\right)=\mathbf{q}_{i}, \quad i=1, \ldots, n .
$$

We only consider such functionals $J(\mathbf{u})$ which can be separated into a sum of similar functionals that only depend on one component $u_{i}$ of the transformation $\mathbf{u}$. Thus, the problem of finding the transformation $\mathbf{u}$ can be subdivided into $d$ problems for each component $z$ of $\mathbf{u}$. In the case of thin-plate spline interpolation the functional $J$ is fully described through the dimension $d$ of the domain and the order $m$ of derivatives used [2]. We will write $J_{m}^{d}$ for these functionals.

$J_{m}^{d}(z)=\sum_{\alpha_{1}+\ldots+\alpha_{d}=m} \frac{m !}{\alpha_{1} ! \cdots \alpha_{d} !} \int_{\mathbb{R}^{d}}\left(\frac{\partial^{m} z}{\partial x_{1}^{\alpha_{1}} \cdots \partial x_{d}^{\alpha_{d}}}\right)^{2} d \mathbf{x}$.

This functional is invariant with respect to translations and rotations, because the integrand is a scalar.

Let a set of functions $\phi_{i}$ span the space $\Pi^{m-1}\left(\mathbb{R}^{d}\right)$ of all polynomials on $\mathbb{R}^{d}$ up to order $m-1$, which is the nullspace of the functional $J_{m}^{d}$. The dimension of this space is

$$
M=\left(\begin{array}{c}
d+m-1 \\
d
\end{array}\right)
$$

and must be lower than $n$ (this gives the minimum number of landmarks). The solution of the minimization problem can now be written in the following form:

$$
z(\mathbf{x})=\sum_{i=1}^{M} a_{i} \phi_{i}(\mathbf{x})+\sum_{i=1}^{n} w_{i} U_{i}(\mathbf{x})
$$

with some basis functions $U_{i}=U\left(\cdot, \mathbf{p}_{i}\right)$ depending on a) the dimension $d$ of the domain, b) the order $m$ of the functional $J$ to be minimized and c) the Hilbert-space $H$ of admissible functions [2]. If we choose the Sobolev-space $H=\mathcal{H}^{2}$, which consists of all square integrable functions, with (weak) derivatives up to second order in $L_{2}(\mathbb{R})$, we obtain the kernel:

$$
U(\mathbf{x}, \mathbf{p})=\left\{\begin{array}{cl}
|\mathbf{x}-\mathbf{p}|^{2 m-d} \ln |\mathbf{x}-\mathbf{p}| & d \text { even } \\
|\mathbf{x}-\mathbf{p}|^{2 m-d} & \text { otherwise }
\end{array}\right.
$$

Note, that the basis functions $U_{i}$ span an $n$-dimensional space of functions that depend only on the source landmarks.

The coefficient vectors $\mathbf{a}=\left(a_{1}, \ldots, a_{M}\right)^{T}$ and $\mathbf{w}=$ $\left(w_{1}, \ldots, w_{n}\right)^{T}$ can be computed through the following system of linear equations:

$$
\begin{aligned}
\mathbf{K w}+\mathbf{P a} & =\mathbf{v} \\
\mathbf{P}^{T} \mathbf{w} & =0
\end{aligned}
$$

where $\mathbf{v}$ is the column vector of one component of the coordinates of the target points $\mathbf{q}_{i}$, and

$$
K_{i j}=U_{i}\left(\mathbf{p}_{j}\right), \quad P_{i j}=\phi_{j}\left(\mathbf{p}_{i}\right) .
$$

\section{Thin-Plate Spline Approximation}

The interpolation approach assumes that the landmark positions are known exactly. In real applications, however, the positions of the landmarks can only be determined approximately. Therefore, in this case an interpolation scheme is inadequate. To take into account the landmark localization errors one has to weaken the interpolation condition (1). This can be done by combining an approximation criterion with the optimization functional (2). In the simplest case of a quadratic approximation term this results in the following functional [3]:

$$
J_{\lambda}(\mathbf{u})=\sum_{i=1}^{n}\left|\mathbf{q}_{i}-\mathbf{u}\left(\mathbf{p}_{i}\right)\right|^{2}+\lambda J_{m}^{d}(\mathbf{u}) .
$$


Such functionals have been used for the reconstruction of surfaces from sparse depth data [4]. For 2D images Arad et al. [5] recently used this approach to represent and modify facial expressions. The first term measures the sum of the quadratic Euclidean distances between the transformed source landmarks and the given target landmarks. We will call this term the data term, because this is the term, where we take into account the data, given by the positions of the corresponding landmarks. The second term measures the smoothness of the resulting transformation. We therefore ask for a transformation $\mathbf{u}(\mathbf{x})$, which i) approximates the displacement of the source landmarks to the target landmarks and ii) is sufficiently smooth. The relative weight between the approximation behavior and the smoothness of the transformation is determined by the parameter $\lambda>0$. If $\lambda$ is small, we obtain a solution with good approximation behavior (in the limit of $\lambda \rightarrow 0$ we have an interpolating transformation). In the other case of a high value for $\lambda$ we obtain a very smooth transformation, with little adaption to the local structure of the distortions determined by the sets of landmarks. In the limit of $\lambda \rightarrow \infty$ we get a global polynom of order up to $m-1$, which has no smoothness energy $J_{m}^{d}$ at all.

The interesting fact is that the solutions to this problem have always the same form as in the case of interpolation given by (3). Also, the computational scheme is nearly the same as in (4). We only have to add the parameter $\lambda$ in the diagonal of the matrix $\mathbf{K}$. Thus $\mathbf{K}$ is substituted by $\mathbf{K}+\lambda \mathbf{I}$.

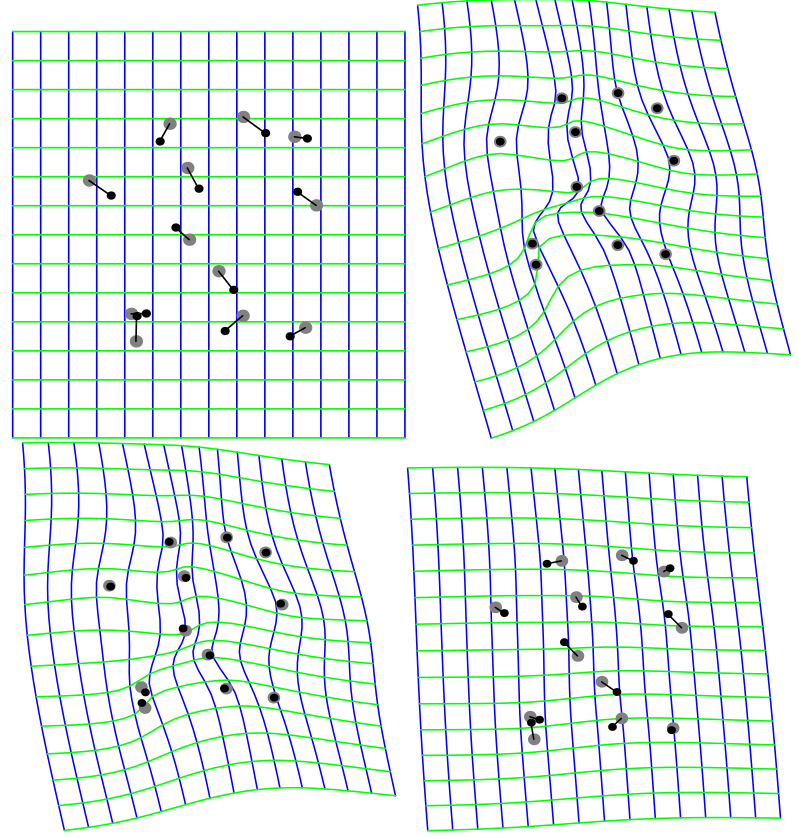

Fig. 1. Thin-plate spline approximation example (parameter: $\lambda=0$, $\lambda=0.001$ and $\lambda=0.1$ ).

Fig. 1 shows an example of image registration using thinplate spline approximation in two dimensions (with $m=2$ ) for different values of the regularization parameter $\lambda$. On the top-left the landmarks are shown on a regular grid. The small black points mark the positions of the source landmarks, the big grey points mark the target landmarks. The other three parts of Fig.1 show the transformed grid after applying the transformation computed through the approximation approach. The top-right part of Fig.1 shows the result for $\lambda=0$, which is equivalent to the interpolation scheme. At some locations the grid is heavily distorted, especially at the two close landmarks in the bottom-left part of the grid. The other values of the regularization parameter are $\lambda=0.001$ (bottom-left) and $\lambda=0.1$ (bottom-right), where the latter one is nearly a pure affine transformation.

Recently, we have applied this approximation method for the registration of tomographic images of the human brain[6]. It turned out that this scheme leads to a more accurate and robust registration result. In particular, outliers do not disturb the result as much as is the case with an interpolation scheme.

The regularization parameter $\lambda$ weights the relevance of the data term with respect to the smoothness condition. A generalization can be made, if we have information about the expected accuracy of the given landmarks. We can weight each single data term $\left|\mathbf{q}_{i}-\mathbf{u}\left(\mathbf{p}_{i}\right)\right|^{2}$ by the inverse variance $1 / \sigma_{i}^{2}$ of the measurement $\mathbf{q}_{i}$. If the variance is high, i.e. if the measurements are uncertain, less penalty is given to the approximation error at this point. With this generalization the data term reads

$$
\sum_{i=1}^{n} \frac{\left|\mathbf{q}_{i}-\mathbf{u}\left(\mathbf{p}_{i}\right)\right|^{2}}{\sigma_{i}^{2}}
$$

And we have to solve the following system of equations:

$$
\begin{aligned}
\left(\mathbf{K}+\lambda \mathbf{W}^{-1}\right) \mathbf{w}+\mathbf{P a} & =\mathbf{v} \\
\mathbf{P}^{T} \mathbf{w} & =0
\end{aligned}
$$

with

$$
\mathbf{W}=\operatorname{diag}\left\{1 / \sigma_{1}^{2}, \ldots, 1 / \sigma_{n}^{2}\right\}
$$

\section{ACKNOWLEDGEMENT}

Support of the Philips Research Laboratories Hamburg, project IMAGINE is gratefully acknowledged.

\section{REFERENCES}

[1] F.L. Bookstein, "Principal warps: Thin-plate splines and the decomposition of deformations," IEEE Trans. on Pattern Analysis and Machine Intelligence, vol. 11, no. 6, pp. 567-585, 1989.

[2] J. Duchon, "Interpolation des fonctions de deux variables suivant le principle de la flexion des plaques minces," R.A.I.R.O. Analyse Numérique, vol. 10, no. 12, 1976.

[3] Grace Wahba, Spline Models for Observational Data, Society for Industrial and Applied Mathematics, Philadelphia, Pennsylvania, 1990.

[4] Richard Szeliski, "Fast surface interpolation using hierachical basis functions," IEEE Trans. on Pattern Analysis and Machine Intelligence, vol. 12 , no. 6 , pp. 513-528, 1990.

[5] N. Arad, N. Dyn, D. Reisfeld, and Y. Yeshurun, "Image warping by radial basis functions: Application to facial expressions," Computer Vision, Graphics, and Image Processing, vol. 56, no. 2, pp. 161-172, 1994 .

[6] K. Rohr, H.S. Stiehl, R.Sprengel, W. Beil, T.M. Buzug, J. Weese, and M.H. Kuhn, "Point-based elastic registration of medical image data using approximating thin-plate splines," Mitteilung FBI-HH-M-254/96, Fachbereich Informatik, Universität Hamburg, 1996. 